Journal of Mathematics and Informatics

Vol. 8, 2017, 83-94

ISSN: 2349-0632 (P), 2349-0640 (online)

Published 29 August 2017

www.researchmathsci.org

DOI: http://dx.doi.org/10.22457/jmi.v8a9

Journal of

Mathematics and

Informatics

\title{
Cone C-class Function with Common Fixed Point Theorems for Cone $b$-metric Space
}

\author{
R. Krishnakumar ${ }^{1}$ and D.Dhamodharan ${ }^{2}$ \\ ${ }^{1}$ Department of Mathematics,Urumu Dhanalakshmi College \\ Tiruchirappalli-620019, India \\ Email: srksacet@yahoo.co.in \\ ${ }^{2}$ Department of Mathematics, Jamal Mohamed College (Autonomous) \\ Tiruchirapplli-620020, India \\ Corresponding author. Email: dharan_raj28@yahoo.co.in \\ Received 1 August 2017; accepted 17 August 2017
}

\begin{abstract}
In this paper, we discuss the common fixed point theorem obtain sufficient conditions for the existence of common fixed points of a pair of mapping satisfying generalized contraction involving rational expressions in cone $b$ metric spaces via cone $C$ class functions
\end{abstract}

Keywords: Common fixed point,cone $b$-metric space,cone metric space cone $C$-class function

AMS Mathematics Subject Classification (2010): 47H10, 54H 25

\section{Introduction and mathematical preliminaries}

In 1089, Bakhtin [2] introduced $b$-metric space as a generalization of metric space. He proved the contraction principle in $b$-metric spaces that generalized the famous contraction principle in metric space. In 2007, Huang and Zhang [4] are introduce the concept of cone metric space and also they discussed some properties of the convergence of sequences and proved the fixed point theorems of a contraction mapping for cone metric spaces; Any mapping $T$ of a complete cone metric space $X$ into itself that satisfies, for some $0 \leq k<1$, the inequality $d(T x, T y) \leq k d(x, y), \forall x, y \in X$ has a unique fixed point. In 2011, Hussain and Saha [6] introduced the concept of cone $b$-metric space as a generalization of $b$-metric spaces and cone metric spaces. They established some topological properties in such spaces and improved some recent results about KKM mappings in the setting of a cone $b$-metric space. Note on $\varphi-\psi$-contractive type mappings and related fixed point are proved by Ansari [11].

In this paper, we investigate the common fixed point theorem obtain sufficient conditions for the existence of common fixed points of a pair of mapping satisfying generalized contraction involving rational expressions in cone $b$ metric spaces via cone $C$ class functions. 


\section{R. Krishnakumar and D.Dhamodharan}

Definition 1.1. Let $E$ be the real Banach space. A subset $P$ of $E$ is called a cone if and only if:

- $P$ is closed, non empty and $P \neq \varnothing$

- $a x+b y \in P$ for all $x, y \in P$ and non negative real numbers $a, b$

- $P \cap(-P)=\{0\}$.

Given a cone $P \subset E$, we define a partial ordering $\leq$ with respect to $\mathrm{P}$ by $x \leq y$ if and only if $y-x \in P$. We will write $x<y$ to indicate that $x \leq y$ but $x \neq y$, while $x, y$ will stand for $y-x \in$ int $P$, where int $P$ denotes the interior of $P$. The cone $P$ is called normal if there is a number $K>0$ such that $0 \leq x \leq y$ implies $\mathrm{P} x \mathrm{P} \leq K \mathrm{P} y \mathrm{P}$ for all $x, y \in E$. The least positive number satisfying the above is called the normal constant.

Example 1.2. [10] Let $K>1$. be given. Consider the real vector space with

$$
\left.E=\left\{a x+b: a, b \in R ; x \in 1-\frac{1}{k}, 1\right]\right\}
$$

with supremum norm and the cone

$$
P=\{a x+b: a \geq 0, b \leq 0\}
$$

in $E$. The cone $P$ is regular and so normal.

Definition 1.3. Let $X$ be a nonempty set. A mapping $d: X \times X \rightarrow E$ is said to be cone metric if and only if, for all $x, y, z \in X$, the following conditions are satisfied:

- $d(x, y)=0$ if and only if $x=y$,

- $d(x, y)=d(y, x)$,

- $d(x, z) \leq d(x, y)+d(y, z)$.

Then $(X, d)$ is called a cone metric space (CMS) .

Example 1.4. Let $E=R^{2}$

$$
P=\{(x, y): x, y \geq 0\}
$$

$X=R$ and $d: X \times X \rightarrow E$ such that

$$
d(x, y)=(|x-y|, \alpha|x-y|)
$$

where $\alpha \geq 0$ is a constant. Then $(X, d)$ is a cone metric space.

Definition 1.5. [6] Let $X$ be a nonempty set and $s \geq 1$ be a given real number. A mapping $d: X \times X \rightarrow E$ is said to be cone $b$-metric if and only if, for all $x, y, z \in X$, the following conditions are satisfied:

- $d(x, y)=0$ if and only if $x=y$,

- $d(x, y)=d(y, x)$,

- $d(x, z) \leq s[d(x, y)+d(y, z)]$.

Then $(X, d)$ is called a cone metric space (CbCMS) . 
Cone C-class Function with Common Fixed Point Theorems for Cone $b$-metric Space

Example 1.6. [5] Let $E=R^{2}$

$$
P=\{(x, y): x, y \geq 0\}
$$

$X=R$ and $d: X \times X \rightarrow E$ such that

$$
d(x, y)=\left(|x-y|^{p}, \alpha|x-y|^{p}\right)
$$

where $\alpha \geq 0$ and $p>1$ are two real constants. Then $(X, d)$ is a cone $b$-metric space with the coefficient $s=2^{p}>1$, but not a cone metric space. In fact, we only need to prove (iii) in Definition 1.5 as follows:

Let $x, y, z \in X$. Set $u=x-z, v=z-y$, so $x-y=u+v$. From the inequality $(a+b)^{p} \leq(2 \max \{a, b\})^{p} \leq 2^{p}\left(a^{p}+b^{p}\right)$ forall $a, b \geq 0$.

we have

$$
|x-y|^{p}=|u+v|^{p} \leq(|u|+|v|)^{p} \leq 2^{p}\left(|u|^{p}+|v|^{p}\right)=2^{p}\left(|x-z|^{p}+|z-y|^{p}\right)
$$

which implies that $d(x, y) \leq s[d(x, z)+d(z, y)]$ with $s=2^{p}>1$. But

$$
|x-y|^{p} \leq|x-z|^{p}+|z-y|^{p}
$$

is impossible for all $x>z>y$. Indeed, taking account of the inequality

$$
(a+b)^{p}>a^{p}+b^{p} \text { forall } a, b \geq 0,
$$

we arrive at

$$
|x-y|^{p}=|u+v|^{p}=(u+v)^{p}>u^{p}+v^{p}=|x-z|^{p}+|z-y|^{p}
$$

for all $\mathrm{x}>\mathrm{z}>\mathrm{y}$. Thus, $\left(\mathrm{c}_{3}\right)$ in Definition 1.3 is not satisfied, i.e., $(\mathrm{X}, \mathrm{d})$ is not a cone metric space.

Example 1.7. [5] Let $X=l^{p}$ with $0<p<1$, where $l^{p}=\left\{\left\{x_{n}\right\} \subset R: \sum_{n=1}^{\infty}\left|x_{n}\right|^{p}<\infty\right\}$.

Let $d: X \times X \rightarrow R_{+}$define by $d(x, y)=\left(\sum_{n=1}^{\infty}\left|x_{n}-y_{n}\right|^{p}\right)^{\frac{1}{p}}$, where $x=\left\{x_{n}\right\}, y=\left\{y_{n}\right\} \in l^{p}$. Then $(X, d)$ is a $b$-metric space [3]. Put $E=l^{1}, P=\left\{\left\{x_{n}\right\} \in E: x_{n} \geq 0\right.$, forall $\left.n \geq 1\right\}$. Letting the mapping $d^{*}: X \times X \rightarrow E$ be defined by $d^{*}(x, y)=\left\{\frac{d(x, y)}{2^{n}}\right\} n \geq 1$, we conclude that $\left(X, d^{*}\right)$ is a cone $b$-metric space with the coefficient $s=2^{\frac{1}{p}}>1$, but it is not a cone metric space.

Definition 1.8. [6] Let $(X, d)$ be a cone b-metric space, $x \in X$ and $\left\{x_{n}\right\}$ be a sequence in $X$. Then

1. $\left\{x_{n}\right\}$ converges to $x$ whenever, for every $c \in E$ with $0=c$, there is a natural number $N$ such that $d\left(x_{n}, x\right)=c$ for all $n \geq N$. We denote this by $\lim _{n \rightarrow \infty} x_{n}=x$ or $x_{n} \rightarrow x(n \rightarrow \infty)$. 
R. Krishnakumar and D.Dhamodharan

2. $\left\{x_{n}\right\}$ is a Cauchy sequence whenever, for every $c \in E$ with $0=c$, there is a natural number $N$ such that $d\left(x_{n}, x_{m}\right)=c$ for all $n, m \geq N$

3. $(X, d)$ is a complete cone $b$-metric space if every Cauchy sequence is convergent.

Definition 1.9. A function $\psi: P \rightarrow P$ is called an altering distance function if the following properties are satisfied:

(i) $\psi$ is non-decreasing and continuous,

(ii) $\psi(t)=0$ if and only if $t=0$.

Definition 1.10. An ultra altering distance function is a continuous, nondecreasing mapping $\varphi: P \rightarrow P$ such that $\varphi(t)>0 \quad, t>0$ and $\varphi(0) \geq 0$.

We denote this set with $\Phi_{u}$

Definition 1.11. [12] A mapping $F: P^{2} \rightarrow P$ is called cone $C$-class function if it is continuous and satisfies following axioms:

1. $F(s, t) \leq s$;

2. $F(s, t)=s$ implies that either $s=0$ or $t=0$; for all $s, t \in P$.

We denote cone $C$-class functions as $\mathrm{C}$.

Example 1.12. [12] The following functions $F: P^{2} \rightarrow P$ are elements of $\mathrm{C}$, for all $s, t \in 0, \infty)$ :

1. $F(s, t)=s-t$,

2. $F(s, t)=k s$, where $0<k<1$,

3. $F(s, t)=s \beta(s)$, where $\beta:[0, \infty) \rightarrow[0,1)$,

4. $\quad F(s, t)=\Psi(s)$, where $\Psi: P \rightarrow P, \Psi(0)=0, \Psi(s)>0$ for all $s \in P$ with $s \neq 0$ and $\Psi(S) \leq s$ for all $s \in P$.,

5. $F(s, t)=s-\varphi(s)$, where $\varphi:[0, \infty) \rightarrow 0, \infty)$ is a continuous function such that $\varphi(t)=0 \Leftrightarrow t=0$;

6. $F(s, t)=s-h(s, t)$, where $h:[0, \infty) \times 0, \infty) \rightarrow 0, \infty)$ is a continuous function such that $h(s, t)=0 \Leftrightarrow t=0$ for all $t, s>0$.

7. $F(s, t)=\varphi(s), F(s, t)=s \Rightarrow s=0$, here $\varphi:[0, \infty) \rightarrow[0, \infty)$ is a upper semi continuous function such that $\varphi(0)=0$ and $\varphi(t)<t$ for $t>0$.

Lemma 1.13. Let $\psi$ and $\varphi$ are altering distance and ultra altering distance functions respectively, $F \in \mathrm{C}$ and $\left\{s_{n}\right\}$ a decreasing sequence in $P$ such that

$$
\psi\left(s_{n+1}\right) \leq F\left(\psi\left(s_{n}\right), \varphi\left(s_{n}\right)\right)
$$

for all $n \geq 1$. Then $\lim _{n \rightarrow \infty} s_{n}=0$. 
Cone C-class Function with Common Fixed Point Theorems for Cone $b$-metric Space

\section{Main result}

Theorem 2.1. Let $(X, d)$ be a complete cone b-metric space (CCbMS) with the co-efficient $s \geq 1$ and $P$ be a normal cone with normal constant $K$. Suppose that the mappings $S, T: X \rightarrow X$ satisfy:

$$
\begin{aligned}
\psi(d(S x, T y)) & \leq F\left(\psi \left(a_{1} d(x, y)+a_{2} \frac{[1+d(x, S x)] d(y, T y)}{1+d(x, y)}+a_{3}[d(x, S x)+d(y, T y)]\right.\right. \\
& \left.+a_{4}[d(x, T y)+d(y, S x)]\right), \varphi\left(a_{1} d(x, y)+a_{2} \frac{[1+d(x, S x)] d(y, T y)}{1+d(x, y)}\right. \\
& \left.\left.+a_{3}[d(x, S x)+d(y, T y)]+a_{4}[d(x, T y)+d(y, S x)]\right)\right)
\end{aligned}
$$

for all $x, y \in X$, where $a_{1}, a_{2}, a_{3}, a_{4}$ are non negative reals with $s a_{1}+a_{2}+(s+1) a_{3}+s(s+1) \quad a_{4}<1 . \psi$ and $\varphi$ are altering distance and ultra altering distance functions respectively, $F \in \mathrm{C}$ such that $\psi(t+s) \leq \psi(t)+\psi(s)$. Then $S$ and $T$ have a unique common fixed point in $X$.

Proof: Let $x_{0}$ be an arbitrary point $X$ and define

$$
\begin{aligned}
& \begin{array}{r}
x_{2 k+1}=S x_{2 k}, x_{2 k+2}=T x_{2 k+1}, k=0,1,2, \cdots \\
\quad \text { Then form } 2.1 \text { we have } \\
\psi\left(\left(x_{2 k+1}, x_{2 k+2}\right)\right) \\
=\psi\left(d\left(S x_{2 k}, T x_{2 k+1}\right)\right) \\
\leq F\left(\psi \left(a_{1} d\left(x_{2 k}, x_{2 k+1}\right)+a_{2} \frac{\left[1+d\left(x_{2 k}, S x_{2 k}\right)\right] d\left(x_{2 k}, T x_{2 k+1}\right)}{1+d\left(x_{2 k}, x_{2 k+1}\right)}\right.\right. \\
\left.+a_{3}\left[d\left(x_{2 k}, S x_{2 k}\right)+d\left(x_{2 k+1} T x_{2 k+1}\right)\right]+a_{4}\left[d\left(x_{2 k}, T x_{2 k+1}\right)+d\left(x_{2 k+1}, S x_{2 k}\right)\right]\right), \\
\quad \varphi\left(a_{1} d\left(x_{2 k}, x_{2 k+1}\right)+a_{2} \frac{\left[1+d\left(x_{2 k}, S x_{2 k}\right)\right] d\left(x_{2 k}, T x_{2 k+1}\right)}{1+d\left(x_{2 k}, x_{2 k+1}\right)}\right. \\
\left.\left.+a_{3}\left[d\left(x_{2 k}, S x_{2 k}\right)+d\left(x_{2 k+1}, T x_{2 k+1}\right)\right]+a_{4}\left[d\left(x_{2 k}, T x_{2 k+1}\right)+d\left(x_{2 k+1}, S x_{2 k}\right)\right]\right)\right) \\
\quad=F\left(\psi \left(a_{1} d\left(x_{2 k}, x_{2 k+1}\right)+a_{2} \frac{\left[1+d\left(x_{2 k}, x_{2 k+1}\right)\right] d\left(x_{2 k}, x_{2 k+2}\right)}{1+d\left(x_{2 k}, x_{2 k+1}\right)}\right.\right. \\
\left.\quad+a_{3}\left[d\left(x_{2 k}, x_{2 k+1}\right)+d\left(x_{2 k+1}, x_{2 k+2}\right)\right]+a_{4}\left[d\left(x_{2 k}, x_{2 k+2}\right)+d\left(x_{2 k+1}, x_{2 k+1}\right)\right]\right), \\
\quad \varphi\left(a_{1} d\left(x_{2 k}, x_{2 k+1}\right)+a_{2} \frac{\left[1+d\left(x_{2 k}, x_{2 k+1}\right)\right] d\left(x_{2 k}, x_{2 k+2}\right)}{1+d\left(x_{2 k}, x_{2 k+1}\right)}\right. \\
\left.\left.\quad+a_{3}\left[d\left(x_{2 k}, x_{2 k+1}\right)+d\left(x_{2 k+1}, x_{2 k+2}\right)\right]+a_{4}\left[d\left(x_{2 k}, x_{2 k+2}\right)+d\left(x_{2 k+1}, x_{2 k+1}\right)\right]\right)\right)
\end{array}
\end{aligned}
$$


R. Krishnakumar and D.Dhamodharan

$$
\begin{aligned}
& =F\left(\psi\left(\left(a_{1}+a_{3}\right) d\left(x_{2 k}, x_{2 k+1}\right)+\left(a_{2}+a_{3}\right) d\left(x_{2 k+1}, x_{2 k+2}\right)+a_{4} d\left(x_{2 k}, x_{2 k+2}\right)\right)\right. \\
& \left.\varphi\left(\left(a_{1}+a_{3}\right) d\left(x_{2 k}, x_{2 k+1}\right)+\left(a_{2}+a_{3}\right) d\left(x_{2 k+1}, x_{2 k+2}\right)+a_{4} d\left(x_{2 k}, x_{2 k+2}\right)\right)\right) \\
& \leq F\left(\psi \left(\left(a_{1}+a_{3}\right) d\left(x_{2 k}, x_{2 k+1}\right)+\left(a_{2}+a_{3}\right) d\left(x_{2 k+1}, x_{2 k+2}\right)\right.\right. \\
& \left.+s a_{4}\left[d\left(x_{2 k}, x_{2 k+1}\right)+d\left(x_{2 k+1}, x_{2 k+2}\right)\right]\right), \varphi\left(\left(a_{1}+a_{3}\right) d\left(x_{2 k}, x_{2 k+1}\right)\right. \\
& \left.\left.+\left(a_{2}+a_{3}\right) d\left(x_{2 k+1}, x_{2 k+2}\right)+s a_{4}\left[d\left(x_{2 k}, x_{2 k+1}\right)+d\left(x_{2 k+1}, x_{2 k+2}\right)\right]\right)\right) \\
& =F\left(\psi\left(\left(a_{1}+a_{3}+s a_{4}\right) d\left(x_{2 k}, x_{2 k+1}\right)+\left(a_{2}+a_{3}+s a_{4}\right) d\left(x_{2 k+1}, x_{2 k+2}\right)\right)\right. \\
& \left.\varphi\left(\left(a_{1}+a_{3}+s a_{4}\right) d\left(x_{2 k}, x_{2 k+1}\right)+\left(a_{2}+a_{3}+s a_{4}\right) d\left(x_{2 k+1}, x_{2 k+2}\right)\right)\right)
\end{aligned}
$$

This implies that $d\left(x_{2 k+1}, x_{2 k+2}\right) \leq \frac{a_{1}+a_{3}+s a_{4}}{1-a_{1}-a_{3}-s a_{4}} d\left(x_{2 k}, x_{2 k+1}\right)$. Similarly, we have

$$
\begin{aligned}
& \psi\left(d\left(x_{2 k+2}, x_{2 k+3}\right)\right) \\
& =\psi\left(d\left(S x_{2 k+1} T x_{2 k+2}\right)\right) \\
& \leq F\left(\psi \left(a_{1} d\left(x_{2 k+1}, x_{2 k+2}\right)+a_{2} \frac{\left[1+d\left(x_{2 k+1}, S x_{2 k+1}\right)\right] d\left(x_{2 k+2}, T x_{2 k+2}\right)}{1+d\left(x_{2 k+1}, x_{2 k+2}\right)}\right.\right. \\
& \left.+a_{3}\left[d\left(x_{2 k+1}, S x_{2 k+1}\right)+d\left(x_{2 k+2,} T x_{2 k+2}\right)\right]+a_{4}\left[d\left(x_{2 k+1} T x_{2 k+2}\right)+d\left(x_{2 k+2}, S x_{2 k+1}\right)\right]\right), \\
& \varphi\left(a_{1} d\left(x_{2 k+1}, x_{2 k+2}\right)+a_{2} \frac{\left[1+d\left(x_{2 k+1}, S x_{2 k+1}\right)\right] d\left(x_{2 k+2}, T x_{2 k+2}\right)}{1+d\left(x_{2 k+1}, x_{2 k+2}\right)}\right. \\
& \left.\left.+a_{3}\left[d\left(x_{2 k+1}, S x_{2 k+1}\right)+d\left(x_{2 k+2,} T x_{2 k+2}\right)\right]+a_{4}\left[d\left(x_{2 k+1} T x_{2 k+2}\right)+d\left(x_{2 k+2}, S x_{2 k+1}\right)\right]\right)\right) \\
& =F\left(\psi \left(a_{1} d\left(x_{2 k+1}, x_{2 k+2}\right)+a_{2} \frac{\left[1+d\left(x_{2 k+1}, x_{2 k+2}\right)\right] d\left(x_{2 k+2}, x_{2 k+3}\right)}{1+d\left(x_{2 k+1}, x_{2 k+2}\right)}\right.\right. \\
& \left.+a_{3}\left[d\left(x_{2 k+1}, x_{2 k+2}\right)+d\left(x_{2 k+2,} x_{2 k+3}\right)\right]+a_{4}\left[d\left(x_{2 k+1}, x_{2 k+3}\right)+d\left(x_{2 k+2}, x_{2 k+2}\right)\right]\right), \\
& \varphi\left(a_{1} d\left(x_{2 k+1}, x_{2 k+2}\right)+a_{2} \frac{\left[1+d\left(x_{2 k+1}, x_{2 k+2}\right)\right] d\left(x_{2 k+2}, x_{2 k+3}\right)}{1+d\left(x_{2 k+1}, x_{2 k+2}\right)}\right. \\
& \left.\left.+a_{3}\left[d\left(x_{2 k+1}, x_{2 k+2}\right)+d\left(x_{2 k+2,} x_{2 k+3}\right)\right]+a_{4}\left[d\left(x_{2 k+1}, x_{2 k+3}\right)+d\left(x_{2 k+2}, x_{2 k+2}\right)\right]\right)\right) \\
& =F\left(\psi\left(\left(a_{1}+a_{3}\right) d\left(x_{2 k+1}, x_{2 k+2}\right)+\left(a_{2}+a_{3}\right) d\left(x_{2 k+2}, x_{2 k+3}\right)+a_{4} d\left(x_{2 k+1}, x_{2 k+3}\right)\right)\right. \text {, } \\
& \left.\varphi\left(\left(a_{1}+a_{3}\right) d\left(x_{2 k+1}, x_{2 k+2}\right)+\left(a_{2}+a_{3}\right) d\left(x_{2 k+2}, x_{2 k+3}\right)+a_{4} d\left(x_{2 k+1}, x_{2 k+3}\right)\right)\right) \\
& \leq F\left(\psi \left(\left(a_{1}+a_{3}\right) d\left(x_{2 k+1}, x_{2 k+2}\right)+\left(a_{2}+a_{3}\right) d\left(x_{2 k+2}, x_{2 k+3}\right)\right.\right. \\
& \left.+s a_{4}\left[d\left(x_{2 k+1}, x_{2 k+2}\right)+d\left(x_{2 k+2}, x_{2 k+3}\right)\right]\right) \text {, } \\
& \varphi\left(\left(a_{1}+a_{3}\right) d\left(x_{2 k+1}, x_{2 k+2}\right)+\left(a_{2}+a_{3}\right) d\left(x_{2 k+2}, x_{2 k+3}\right)\right. \\
& \left.\left.+s a_{4}\left[d\left(x_{2 k+1}, x_{2 k+2}\right)+d\left(x_{2 k+2}, x_{2 k+3}\right)\right]\right)\right) \\
& =F\left(\psi\left(\left(a_{1}+a_{3}+s a_{4}\right) d\left(x_{2 k+1}, x_{2 k+2}\right)+\left(a_{2}+a_{3}+s a_{4}\right) d\left(x_{2 k+2}, x_{2 k+3}\right)\right),\right. \\
& \left.\varphi\left(\left(a_{1}+a_{3}+s a_{4}\right) d\left(x_{2 k+1}, x_{2 k+2}\right)+\left(a_{2}+a_{3}+s a_{4}\right) d\left(x_{2 k+2}, x_{2 k+3}\right)\right)\right) \text {. }
\end{aligned}
$$

This implies that

$$
d\left(x_{2 k+2}, x_{2 k+3}\right) \leq \frac{a_{1}+a_{3}+s a_{4}}{1-a_{1}-a_{3}-s a_{4}} d\left(x_{2 k+1}, x_{2 k+2}\right) .
$$


Cone C-class Function with Common Fixed Point Theorems for Cone $b$-metric Space

Putting $\lambda=\frac{a_{1}+a_{3}+s a_{4}}{1-a_{1}-a_{3}-s a_{4}}$

As $s a_{1}+a_{2}+(s+1) a_{3}+s(s+1) a_{4}<1$, it is clear that $<1 / s$, we have,

$$
d\left(x_{n+1}, x_{n+2}\right) \leq \lambda\left(x_{n}, x_{n+1}\right) \leq \cdots \leq \lambda^{n+1} d\left(x_{0}, x_{1}\right) .
$$

Hence for any $m>n$, we have

$$
\begin{aligned}
d\left(x_{n}, x_{m}\right) & \leq s\left[d\left(x_{n}, x_{n+1}\right)+d\left(x_{n+1}, x_{m}\right)\right] \\
& =s d\left(x_{n}, x_{n+1}\right)+s d\left(x_{n+1}, x_{m}\right) \\
& \leq s d\left(x_{n}, x_{n+1}\right)+s^{2}\left[d\left(x_{n+1}, x_{n+2}\right)+d\left(x_{n+2}, x_{m}\right)\right] \\
& =s d\left(x_{n}, x_{n+1}\right)+s^{2} d\left(x_{n+1}, x_{n+2}\right)+s^{2} d\left(x_{n+2}, x_{m}\right) \\
& \leq s d\left(x_{n}, x_{n+1}\right)+s^{2} d\left(x_{n+1}, x_{n+2}\right)+s^{3} d\left(x_{n+2}, x_{n+3}\right)+\cdots+s^{n+m-1} d\left(x_{n+m-1}, x_{m}\right) \\
& \leq s \lambda^{n} d\left(x_{1}, x_{0}\right)+s^{2} \lambda^{n+1} d\left(x_{1}, x_{0}\right)+s^{3} \lambda^{n+2} d\left(x_{1}, x_{0}\right)+\cdots+s^{m} \delta^{n+m-1} d\left(x_{1}, x_{0}\right) \\
& =s \lambda^{n}\left[1+s \lambda+s^{2} \lambda^{2}+s^{3} \lambda^{3}+\cdots+(s \lambda)^{m-1}\right] d\left(x_{1}, x_{0}\right) \\
& \leq\left[\frac{s \lambda^{n}}{1-s \lambda}\right] d\left(x_{1}, x_{0}\right) .
\end{aligned}
$$

Since $\mathrm{P}$ is a normal cone with normal constant $\mathrm{K}$, so we get

$\mathrm{P} d\left(x_{n}, x_{m}\right) \mathrm{P} \leq K \frac{s \lambda^{n}}{1-s \lambda} \mathrm{P} d\left(x_{1}, x_{0}\right) \mathrm{P}$.

This implies $\mathrm{P} d\left(x_{n}, x_{m}\right) \mathrm{P} \rightarrow 0$ as $n, m \rightarrow \infty$ since $0<s \lambda<1$.

Hence $\left\{x_{n}\right\}$ is a Cauchy sequence. Since $(X, d)$ is a complete cone b-metric space, there exists $p \in X$ such that $x_{n} \rightarrow p$ as $n \rightarrow \infty$. Now, since

$$
\begin{aligned}
& \psi(d(p, T p)) \leq \psi\left(s\left[d\left(p, x_{2 n+1}\right)+d\left(x_{2 n+1}, T p\right)\right]\right) \\
& =\psi\left(s d\left(S x_{2 n}, T p\right)+s d\left(p, x_{2 n+1}\right)\right) \\
& =\psi\left(s d\left(S x_{2 n}, T p\right)\right)+\psi\left(s d\left(p, x_{2 n+1}\right)\right) \\
& \leq F\left(\psi \left(s d\left(p, x_{2 n+1}\right)+s\left[a_{1} d\left(x_{2 n}, p\right)+a_{2} \frac{\left[1+d\left(x_{2 n}, S x_{2 n}\right)\right] d(p, T p)}{1+d\left(x_{2 n}, p\right)}\right.\right.\right. \\
& \left.\left.+a_{3}\left(d\left(x_{2 n}, x_{2 n+1}\right)+d(p, T p)\right)+a_{4}\left(d\left(x_{2 n}, T p\right)+d\left(p, S x_{2 n}\right)\right)\right]\right), \\
& \left(s d\left(p, x_{2 n+1}\right)+s\left[a_{1} d\left(x_{2 n}, p\right)+a_{2} \frac{\left[1+d\left(x_{2 n}, S x_{2 n}\right)\right] d(p, T p)}{1+d\left(x_{2 n}, p\right)}\right.\right. \\
& \left.\left.\left.+a_{3}\left(d\left(x_{2 n}, x_{2 n+1}\right)+d(p, T p)\right)+a_{4}\left(d\left(x_{2 n}, T p\right)+d\left(p, S x_{2 n}\right)\right)\right]\right)\right)+\psi\left(s d\left(p, x_{2 n+1}\right)\right) \\
& =F\left(\psi \left(s d\left(p, x_{2 n+1}\right)+s\left[a_{1} d\left(x_{2 n}, p\right)+a_{2} \frac{\left[1+d\left(x_{2 n}, x_{2 n+1}\right)\right] d(p, T p)}{1+d\left(x_{2 n}, p\right)}\right.\right.\right. \\
& \left.\left.+a_{3} d\left(x_{2 n}, x_{2 n+1}+d(p, T p)\right)+a_{4}\left(d\left(x_{2 n}, T p\right)+d\left(p, S x_{2 n}\right)\right)\right]\right),
\end{aligned}
$$


R. Krishnakumar and D.Dhamodharan

$$
\begin{aligned}
& \varphi\left(\left(s d\left(p, x_{2 n+1}\right)+s\left[a_{1} d\left(x_{2 n}, p\right)+a_{2} \frac{\left[1+d\left(x_{2 n}, x_{2 n+1}\right)\right] d(p, T p)}{1+d\left(x_{2 n}, p\right)}\right.\right.\right. \\
& \left.\left.\left.\left.+a_{3} d\left(x_{2 n}, x_{2 n+1}+d(p, T p)\right)+a_{4}\left(d\left(x_{2 n}, T p\right)+d\left(p, S x_{2 n}\right)\right)\right]\right)\right)\right)+\psi\left(s d\left(p, x_{2 n+1}\right)\right) \\
& \leq F\left(\psi \left(s d\left(p, x_{2 n+1}\right)+s\left[a_{1} d\left(x_{2 n}, p\right)+a_{2} \frac{\left[1+d\left(x_{2 n}, x_{2 n+1}\right)\right] d(p, T p)}{1+d\left(x_{2 n}, p\right)}\right.\right.\right. \\
& \left.\left.+a_{3} d\left(x_{2 n}, x_{2 n+1}+d(p, T p)\right)+a_{4}\left(d\left(x_{2 n}, T p\right)+d\left(p, S x_{2 n}\right)\right)\right]\right), \\
& \varphi\left(\left(s d\left(p, x_{2 n+1}\right)+s\left[a_{1} d\left(x_{2 n}, p\right)+a_{2} \frac{\left[1+d\left(x_{2 n}, x_{2 n+1}\right)\right] d(p, T p)}{1+d\left(x_{2 n}, p\right)}\right.\right.\right. \\
& \left.\left.\left.\left.+a_{3} d\left(x_{2 n}, x_{2 n+1}+d(p, T p)\right)+a_{4}\left(d\left(x_{2 n}, T p\right)+d\left(p, S x_{2 n}\right)\right)\right]\right)\right)\right)
\end{aligned}
$$

As $x_{n} \rightarrow p$ and $x_{n+1} \rightarrow p$ as $n \rightarrow \infty$, we get

$\left(1-s a_{2}-s a_{3}-s a_{4}\right) \mathrm{P} d(p, T p) \mathrm{P} \leq K\left[s a_{1} \mathrm{P} d\left(x_{2 n}, p\right) \mathrm{P}+s\left(a_{4}\right) \mathrm{P} d\left(p, x_{2 n+1}\right) \mathrm{P}\right] \rightarrow 0$ as $n \rightarrow \infty$.

Hence $\mathrm{P} d(T p, p) \mathrm{P}=0$. Since $\left(1-s a_{2}-s a_{3}-s a_{4}\right)>0$.

Thus we get $T p=p$, that is, $p$ is a fixed point of $T$.

Uniqueness:

Let $q$ be another fixed point common to $S$ and $T$, that is $S q=T q=q$ such that $p \neq q$.

Then from 2.1 we have $\psi(d(p, q))$

$$
\begin{aligned}
& =\psi(d(S p, T p)) \\
& \leq F\left(\psi \left(a_{1} d(p, T p)+a_{2} \frac{[1+d(p, s p)] d(q, T p)}{1+d(p, q)}+a_{3}[d(p, S p)+d(q, T p)]\right.\right. \\
& \left.+a_{4}(d(p, T q)+d(q, S p))\right), \varphi\left(a_{1} d(p, T p)+a_{2} \frac{[1+d(p, s p)] d(q, T p)}{1+d(p, q)}\right. \\
& \left.\left.+a_{3}[d(p, S p)+d(q, T p)]+a_{4}(d(p, T q)+d(q, S p))\right)\right) \\
& =F\left(\psi \left(a_{1} d(p, q)+a_{2} \frac{[1+d(p, p)] d(q, q)}{1+d(p, q)}+a_{3}[d(p, p)+d(q, q)]\right.\right. \\
& \left.+a_{4}(d(p, q)+d(q, p))\right), \\
& \varphi\left(a_{1} d(p, q)+a_{2} \frac{[1+d(p, p)] d(q, q)}{1+d(p, q)}+a_{3}[d(p, p)+d(q, q)]\right. \\
& \left.\left.+a_{4}(d(p, q)+d(q, p))\right)\right) \\
& =F\left(\psi\left(\left(a_{1}+2 a_{4}\right) d(p, q)\right), \varphi\left(\left(a_{1}+2 a_{4}\right) d(p, q)\right)\right) \\
& \leq F\left(\psi\left(\left(s a_{1}+a_{2}+(s+1) a_{3}+s(s+1) a_{4}\right) d(p, q)\right)\right. \\
& \left.\varphi\left(\left(s a_{1}+a_{2}+(s+1) a_{3}+s(s+1) a_{4}\right) d(p, q)\right)\right) \\
& <\psi(d(p, q))
\end{aligned}
$$


Cone C-class Function with Common Fixed Point Theorems for Cone $b$-metric Space which is a contradiction. Hence $\mathrm{Pd}(p, q) \mathrm{P}=0$ and so $p=q$. Thus $p$ is a unique common fixed point of $S$ and $T$.

This completes the proof. Putting $S=T$, we have the following results.

Corollary 2.2. Let $(X, d)$ be a complete cone b-metric space (CCbMS) with the co-efficient $s \geq 1$ and $P$ be a normal cone with normal constant $K$. Suppose that the mappings $S, T: X \rightarrow X$ satisfy:

$$
\begin{aligned}
\psi(d(T x, T y)) & \leq F\left(\psi \left(a_{1} d(x, y)+a_{2} \frac{[1+d(x, T x) d(y, T y)]}{1+d(x, y)}+a_{3}[d(x, T x)+d(y, T y)]\right.\right. \\
& \left.+a_{4}[d(x, T y)+d(y, T x)]\right), \varphi\left(a_{1} d(x, y)+a_{2} \frac{[1+d(x, T x) d(y, T y)]}{1+d(x, y)}\right. \\
& \left.\left.+a_{3}[d(x, T x)+d(y, T y)]+a_{4}[d(x, T y)+d(y, T x)]\right)\right)
\end{aligned}
$$

for all $x, y \in X$, where $a_{1}, a_{2}, a_{3}, a_{4}$ are non negative reals with $s a_{1}+a_{2}+(s+1) a_{3}+s(s+1) a_{4}<1 . \psi$ and $\varphi$ are altering distance and ultra altering distance functions respectively, $F \in \mathrm{C}$ such that $\psi(t+s) \leq \psi(t)+\psi(s)$. Then $T$ has a unique common fixed point in $X$.

Proof: The proof of corollary (2.2) immediately follows from Theorem (2.1) by taking $S=T$. This completes the proof.

Corollary 2.3. Let $(X, d)$ be a complete cone b-metric space (CCbMS) with the co-efficient $s \geq 1$ and $P$ be a normal cone with normal constant $K$. Suppose that the mappings $S, T: X \rightarrow X$ satisfy:

$$
\begin{aligned}
& \psi\left(d\left(T^{n} x, T^{n} y\right)\right) \\
& \quad \leq F\left(\psi \left(a_{1} d(x, y)+a_{2} \frac{\left[1+d\left(x, T^{n} x\right) d\left(y, T^{n} y\right)\right]}{1+d(x, y)}+a_{3}\left[d\left(x, T^{n} x\right)+d\left(y, T^{n} y\right)\right]\right.\right. \\
& \left.\quad+a_{4}\left[d\left(x, T^{n} y\right)+d\left(y, T^{n} x\right)\right]\right), \varphi\left(a_{1} d(x, y)+a_{2} \frac{\left[1+d\left(x, T^{n} x\right) d\left(y, T^{n} y\right)\right]}{1+d(x, y)}\right. \\
& \left.\left.\quad+a_{3}\left[d\left(x, T^{n} x\right)+d\left(y, T^{n} y\right)\right]+a_{4}\left[d\left(x, T^{n} y\right)+d\left(y, T^{n} x\right)\right]\right)\right)
\end{aligned}
$$

for all $x, y \in X$, where $a_{1}, a_{2}, a_{3}, a_{4}$ are non negative reals with $s a_{1}+a_{2}+(s+1) a_{3}+s(s+1) a_{4}<1 . \psi$ and $\varphi$ are altering distance and ultra altering distance functions respectively, $F \in \mathrm{C}$ such that $\psi(t+s) \leq \psi(t)+\psi(s)$. Then $T$ has a unique common fixed point in $X$.

Proof: By Corollary (2.2) there exists $u \in X$ such that $T^{n} u=u$. Then 
R. Krishnakumar and D.Dhamodharan

$$
\begin{aligned}
\psi(d(T u, u)) & =\psi\left(d\left(T T^{n} u, T^{n} u\right)\right) \\
& =\psi\left(d\left(T^{n} T u, T^{n} u\right)\right) \\
& \leq F\left(\psi \left(a_{1} d(T u, u)+a_{2} \frac{\left[1+d\left(T u, T^{n} T u\right)\right] d\left(u, T^{n} u\right)}{1+d(T u, u)}\right.\right. \\
& \left.+a_{3}\left[d\left(T u, T^{n} T u\right)+d\left(u, T^{n} u\right)\right]+a_{4}\left[d\left(T u, T^{n} u\right)+d\left(u, T^{n} T u\right)\right]\right), \\
& \varphi\left(a_{1} d(T u, u)+a_{2} \frac{\left[1+d\left(T u, T^{n} T u\right)\right] d\left(u, T^{n} u\right)}{1+d(T u, u)}\right. \\
& \left.\left.+a_{3}\left[d\left(T u, T^{n} T u\right)+d\left(u, T^{n} u\right)\right]+a_{4}\left[d\left(T u, T^{n} u\right)+d\left(u, T^{n} T u\right)\right]\right)\right) \\
& \leq F\left(\psi \left(a_{1} d(T u, u)+a_{2} \frac{\left[1+d\left(T u, T T^{n} u\right)\right] d\left(u, T^{n} u\right)}{1+d(T u, u)}\right.\right. \\
& \left.+a_{3}\left[d\left(T u, T T^{n} u\right)+d\left(u, T^{n} u\right)\right]+a_{4}\left[d\left(T u, T^{n} u\right)+d\left(u, T T^{n} u\right)\right]\right), \\
& \varphi\left(a_{1} d(T u, u)+a_{2} \frac{\left[1+d\left(T u, T T^{n} u\right)\right] d\left(u, T^{n} u\right)}{1+d(T u, u)}\right. \\
& \left.\left.+a_{3}\left[d\left(T u, T T^{n} u\right)+d\left(u, T^{n} u\right)\right]+a_{4}\left[d\left(T u, T^{n} u\right)+d\left(u, T T^{n} u\right)\right]\right)\right) \\
& =F\left(\psi \left(a_{1} d(T u, u)+a_{2} \frac{[1+d(T u, T u)] d(u, u)}{1+d(T u, u)}\right.\right. \\
& \left.+a_{3}[d(T u, T u)+d(u, u)]+a_{4}[d(T u, u)+d(u, T u)]\right), \\
& \varphi\left(a_{1} d(T u, u)+a_{2} \frac{[1+d(T u, T u)] d(u, u)}{1+d(T u, u)}\right. \\
& \left.\left.+a_{3}[d(T u, T u)+d(u, u)]+a_{4}[d(T u, u)+d(u, T u)]\right)\right) \\
& =\psi\left(\left(a_{1}+2 a_{4}\right) d(T u, u)\right)
\end{aligned}
$$

and so $d(T u, u)=0$. Thus, $T u=u$. This show that $T$ has a unique fixed point $X$.

This completes the proof.

Putting $a_{1}=k, a_{2}=a_{3}=a_{4}=0$ in Corollary (2.2) then we have the following result.

Corollary 2.4. Let $(X, d)$ be a complete cone b-metric space (CCbMS) with the co-efficient $s \geq 1$ and $P$ be a normal cone with normal constant $K$. Suppose that the mappings $S, T: X \rightarrow X$ satisfy:

$$
\psi(d(T x, T y)) \leq F(\psi(k d(x, y)), \varphi(k d(x, y)))
$$

For all $x, y \in X$, where $k \in(0,1)$ is a constant with $s k<1 . \psi$ and $\varphi$ are altering distance and ultra altering distance functions respectively, $F \in \mathrm{C}$ such that $\psi(t+s) \leq \psi(t)+\psi(s)$. Then $T$ has a unique common fixed point in $X$.

Note 2.5. Corollary 2.4 extends well known Banach contraction principle from complete metric space to that setting of complete cone $b$-metric space via cone $\mathrm{C}$-class function consider in this paper.

Putting $a_{3}=k, a_{1}=a_{2}=a_{4}=0$ in corollary (2.2), then we have the following result. 
Cone C-class Function with Common Fixed Point Theorems for Cone $b$-metric Space

Corollary 2.6. Let $(X, d)$ be a complete cone b-metric space (CCbMS) with the co-efficient $s \geq 1$ and $P$ be a normal cone with normal constant $K$. Suppose that the mappings $S, T: X \rightarrow X$ satisfy:

$$
\psi(d(T x, T y)) \leq F(\psi(k[d(x, T x)+d(y, T y)]), \varphi(k[d(x, T x)+d(y, T y)]))
$$

For all $x, y \in X$, where $k \in\left(0, \frac{1}{s+1}\right)$ is a constant with $k(s+1)<1 . \psi$ and $\varphi$ are altering distance and ultra altering distance functions respectively, $F \in \mathrm{C}$ such that $\psi(t+s) \leq \psi(t)+\psi(s)$. Then $T$ has a unique common fixed point in $X$.

Note 2.7. Corollary 2.6 extends well known Kannan contraction principle from complete metric space to that setting of complete cone $b$-metric space via cone $\mathrm{C}$-class function consider in this paper.

Putting $a_{4}=k, a_{1}=a_{2}=a_{3}=0$ in corollary (2.2), then we have the following result.

Corollary 2.8. Let $(X, d)$ be a complete cone b-metric space (CCbMS) with the co-efficient $s \geq 1$ and $P$ be a normal cone with normal constant $K$. Suppose that the mappings $S, T: X \rightarrow X$ satisfy:

$$
\psi(d(T x, T y)) \leq F(\psi(k[d(x, T y)+d(y, T x)]), \varphi(k[d(x, T y)+d(y, T x)]))
$$

For all $x, y \in X$, where $k \in\left(0, \frac{1}{s+1}\right)$ is a constant with $k(s+1)<1 . \psi$ and $\varphi$ are altering distance and ultra altering distance functions respectively, $F \in \mathrm{C}$ such that $\psi(t+s) \leq \psi(t)+\psi(s)$. Then $T$ has a unique common fixed point in $X$.

Note 2.9. Corollary 2.8 extends well known Kannan contraction principle from complete metric space to that setting of complete cone $b$-metric space via cone $\mathrm{C}$-class function consider in this paper.

\section{REFERENCES}

1. T.Abdeljawad, D.Turkloglu and M.Abuloha, Some theorems and examples of cone metric spaces, J. Comput. Anal. Appl., 12(4) (2010) 739-753.

2. I.A.Bakhtin, The contraction mapping principle in almost metric space, Funct. Anal. Gos. Ped. Inst. Unianowsk, 30 (1989) 26-37.

3. M.Boriceanu, M.Bota and A.Petrusel, Mutivalued fractals in $b$-metric spaces, Cent. Eur. J. Math., 8(2) (2010) 367-377.

4. L.G.Huang and Zhang, Cone metric spaces and fixed point theorems of contractive mappings, J. Math. Anal. Appl., 332 (2007) 1468-1476.

5. Huang.H, S. Xu, Fixed point theorems of contractive mappings in cone $b$ metric spaces and applications, Fixed point Theory Appl., 112 (2013)

6. N. Hussain, MH. Shah, KKM mapping in cone $b$-metric spaces, Comput. Math. Appl., 62 (2011) 1677-1684.

7. R.Krishnakumar and M.Marudai, Cone convex metric space and fixed point theorems, 


\section{R. Krishnakumar and D.Dhamodharan}

Int. Journal of Math. Analysis, 6(22) (2012) 1087-1093.

8. R.Krishnakumar and D.Dhamodharan, Common fixed point of four mapping with contractive modulus on cone banach space, Malaya J. Mat., 5(2) (2017) 310-320.

9. R.Krishnakumar and D.Dhamodharan, Fixed point theorems in normal cone metric space, International J. of Math. Sci. \& Engg. Appls. 10(III) (2016) 213-224.

10. M.Asadi and H.Soleimani, Examples in cone metric spaces: a survey, Middle -East Journal of Scientific Research ,11(12) (2012) 1636-1640.

11. A.H.Ansari, Note on $\varphi-\psi$-contractive type mappings and related fixed point, The 2nd Regional Conference on Mathematics and Applications, PNU, September 2014, 377-380.

12. A.H.Ansari, S.Chandok, N.Hussin and L.Paunovic, Fixed points of $(\psi, \phi)$ - weak contractions in regular cone metric spaces via new function, J. Adv. Math. Stud., 9(1) (2016) 72-82. 